\title{
Genomics of non-photosynthetic plants
}

Logacheva M.D. ${ }^{1,2 *}$

${ }^{1}$ Skolkovo Institute of Science and Technology, Moscow, Russia

${ }^{2}$ Lomonosov Moscow State University, Institute of Physico-Chemical Biology, Moscow, Russia

*maria.log@gmail.com

The capability to photosynthesis is one of the most prominent characteristics of plants. However, several species have lost this ability and have adapted to obtain energy from organic compounds derived from other organisms: either from other plants (parasitism) or from fungi (mycoheterotrophy). Heterotrophy is not confined to any specific lineage of plants; it has occurred repeatedly in the course of evolution. The switch to heterotrophy leads to profound changes at the phenotypic level (reduction of leaves, loss of green colour, reduction of the vegetation period) that are highly parallel in different lineages. Heterotrophic plants are difficult to cultivate in experimental conditions; this hampers classic genetic and physiological studies. Advances in DNA sequencing technologies permit the application of a genomic approach for elucidation the genetic changes associated with heterotrophy. I will summarize recent discoveries on the genomics of nonphotosynthetic plants, with focus on the following aspects: 1) structure and evolution of organellar genomes, 2) loss and expansion of specific gene families, 3) horizontal gene transfer, 4) mycoheterotrophy and parasitism - differences and similarities at genomic level. 